\title{
A case of abdominal pain and fear of intravenous contrast
}

\author{
Bader Hammami $\mathrm{M}^{1,2 *}$, Pratik Pandit ${ }^{1}$ and Adam J Fritz ${ }^{1}$ \\ ${ }^{1}$ Department of Internal Medicine, Saint Louis University School of Medicine, St. Louis, MO, USA \\ ${ }^{2}$ Division of Gastroenterology and Hepatology, Saint Louis University School of Medicine, St. Louis, MO, USA
}

\section{Case presentation}

A 66-year-old male with a past medical history of 50-pack-year tobacco smoking and atrial fibrillation presented to an outside facility with abdominal pain and hematochezia. Of note, he was not taking his home medications of Metoprolol and Coumadin for a few weeks due to lack of insurance. He was found to have lactic acidosis, acute kidney injury, and significant leukocytosis. The patient was transferred to our facility for further evaluation and management.

On presentation to our ED, the patient was in atrial fibrillation with a rate of 104 . He reaffirmed his story and provided additional history, including chronic symptoms suggestive of claudication. A nonenhanced CT of the abdomen and pelvis was obtained in the ED and revealed mural thickening of a jejunal loop.

After evaluation by the vascular surgery service, further contrasted imaging was deferred due to acute renal failure. An ultrasound of the abdominal vessels was performed. The preliminary interpretation was deemed to be inconclusive, and the patient was followed clinically on the medicine service.

Over the following 24 hours, the patient developed acute neurovascular changes in the bilateral lower extremities, with total paralysis and sensory changes. Emergent CT angiogram (Figure 1) showed complete abrupt occlusion of the aorta just above the level of the diaphragmatic crus with non opacification of the abdominal

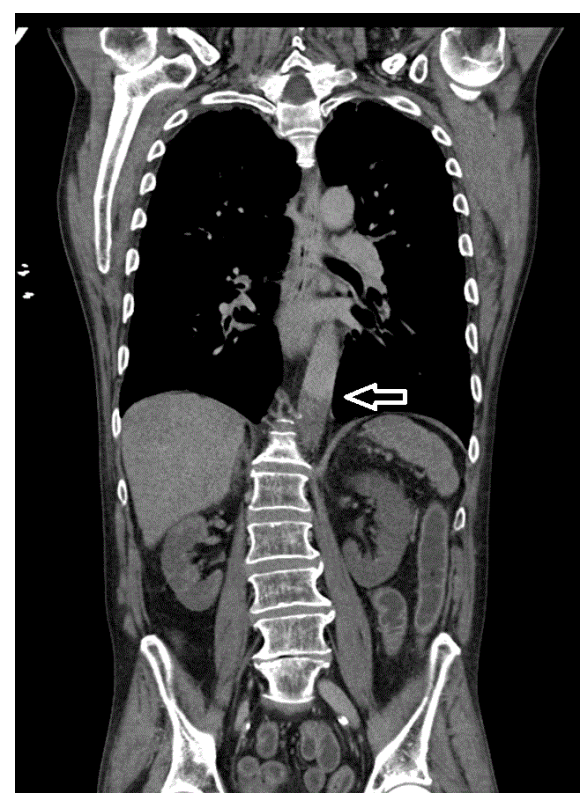

Figure 1. Acute aortic occlusion. aorta and its branches extending to the iliac arteries and evidence of renal and bowel ischemia. The patient was then taken emergently to the OR for aortic thrombectomy. The extent of his vascular insult was significant, and the patient ultimately passed away. It was only after the events of the surgery that a formal read on the ultrasound revealed that the patient had no blood flow within the aorta and branch vessels.

\section{Discussion and teachable moment}

Acute mesenteric ischemia has persisted as a source of mortality across several decades [1]. The etiology of mesenteric ischemia is commonly associated with cardio-embolic events and native vascular disease. However the cause of ischemia in this case was a massive occlusion of the abdominal aorta, which is unusual [2]. Occlusion of the aorta can be seen in large abdominal aortic aneurysms, and rarely from embolic events.

Despite absent aortic flow on ultrasound, our patient did not exhibit symptoms of aortic occlusion for several hours, resulting in a delay of surgical intervention. It was not until CT angiography was performed that assurance of the patient's diagnosis was made and surgical intervention was enacted. We believe our patient suffered a thromboembolic event from a cardiogenic source, given his history of atrial fibrillation without anti-coagulation. However, acute thrombosis is possible, given identified atherosclerosis on imaging, smoking history, and description of chronic symptoms. While a cardiac thrombus would typically go to the brain, a large thrombus, as presumed in this case, could bypass the brachiocephalic arteries and progress through the aorta.

Our case highlights several important points. The first, being that in spite of a thorough history and an abundance of clinical data, the critical piece of data for making the diagnosis of mesenteric ischemia is a contrasted CT scan. A meta-analysis examining the diagnosis of acute mesenteric ischemia found that among all of the data collected when working towards a diagnosis of acute mesenteric ischemia, the CT angiogram was the most important and definitive [3]. Accordingly, it would seem that no argument would be raised regarding the use of contrasted imaging in the evaluation of acute mesenteric ischemia. However, administration of contrast in patients with acute renal insufficiency is usually a source of pause for discussion.

Correspondence to: Muhammad B Hammami, Gastroenterology and Hepatology, Department of Internal Medicine, Saint Louis University School of Medicine, 1402 S. Grand Blvd., St Louis, MO 63104, USA, Tel: +1 3145778764 , Fax: +1 3145778125 E-mail: mbhammami@hotmail.com

Received: April 02, 2017; Accepted: April 25, 2017; Published: April 29, 2017 
Thus, another important point of discussion is the administration of contrast in patients with acute renal insufficiency. Ultimately, contrast administration should not be delayed if the severity of illness outweighs the risk of contrast-induced nephropathy. There are many strategies available to clinicians to mitigate the risk of contrastinduced nephropathy. However, studies have shown that these techniques (pre-hydration with intravenous fluids and administration of $\mathrm{N}$-acetylcysteine) are inconsistently used in practice [4]. A systematic review and meta-analysis of contrast induced acute kidney injury showed no difference between patients who did and did not receive contrast, regardless of previous renal insufficiency, making the withholding of contrasted imaging unnecessary [5]. As such, the question of whether contrast poses a threat to an individual patient must be weighed on a case-by-case basis. Had the CT angiography been obtained prior to symptom development of acute aortic occlusion in our patient, it raises the question of if the patient's outcome would have been different.
Clinical context, knowledge of preventative practices, and ultimate risk of contrast induced nephropathy should help guide clinicians when in doubt with regards to utilizing CT angiogram in the diagnosis of acute mesenteric ischemia. Perhaps simple occlusion of the superior mesenteric artery or its branches may be the source of ischemia, or perhaps a more catastrophic etiology will be uncovered.

\section{References}

1. Sise MJ (2014) Acute mesenteric ischemia. Surg Clin North Am 94: 165-181.

2. Bogie R, Willigendael EM, De booij M, Meesters B, Teijink JA (2008) Acute thrombosis of an abdominal aortic aneurysm: a short report. Eur J Vasc Endovasc Surg 35: 590-592. [Crossref]

3. Cudnik MT, Darbha S, Jones J, Macedo J, Stockton SW, et al. (2013) The diagnosis of acute mesenteric ischemia: A systematic review and meta-analysis. Acad Emerg Med 20: 1087-1100. [Crossref]

4. Weisbord SD, Mor MK, Resnick AL (2008) Prevention, incidence, and outcomes of contrast-induced acute kidney injury. Arch Intern Med 168: 1325-1332. [Crossref]

5. Mcdonald JS, Mcdonald RJ, Comin J (2013) Frequency of acute kidney injury following intravenous contrast medium administration: a systematic review and metaanalysis. Radiology 267: 119-28. [Crossref]

Copyright: (C2017 Hammami BM. This is an open-access article distributed under the terms of the Creative Commons Attribution License, which permits unrestricted use, distribution, and reproduction in any medium, provided the original author and source are credited. 